\title{
PReS-FINAL-2058: Lipid peroxides, lipophuscin, sulfhydryl groups and TOS in children with JIA
}

\author{
H Mazur Zielinska ${ }^{1 *}$, M Zielinski ${ }^{1}$ D Karbowska², L Pilarz², E Birkner ${ }^{3}$ \\ From 20th Pediatric Rheumatology European Society (PReS) Congress \\ Ljubljana, Slovenia. 25-29 September 2013
}

\section{Introduction}

The etiology of most joints' inflammations is unknown. Arthritis in children can have a diversified course. Various parameters useful in diagnosis and treatment of different forms of inflammations of the joints are being researched. Reactive oxygen species in large concentrations are toxic and cause, among others, the phenomenon of polyunsaturated fatty acids lipid peroxidation, which results in the formation of aldehyde compounds. Non enzymatic antioxidants include: lipid peroxides $(\mathrm{LHP}=\mathrm{LOOH})$, lipophuscin (LF). An important part of non enzymatic antioxidative defense are sulfhydryl groups-SH. Total oxidative status (TOS) is used as an indicator of the overall oxidative potential of cells.

\section{Objectives}

The aim of the study was to find how the level of the selected oxidative parameters changes in serum in children with inflammation of the joints. The correlation between the selected oxidative parameters and disease's relapses is also studied.

\section{Methods}

The studied parameters were measured in blood serum of 59 patients with JIA, aged from 2 to 18 years old, hospitalized in the Rheumatology Division of the Department of Pediatrics, Silesian Medical University. The control group consisted of 25 healthy children.

\section{Results}

See Table 1.

\section{Conclusion}

The studied parameters of oxidative status differ between children with arthritis and healthy ones. Lipid
Table 1

\begin{tabular}{lll}
\hline & JIA & Control group \\
\hline SH & $246,7 \pm 53,81$ & $329,8 \pm 24,23$ \\
\hline LF & $741,2 \pm 208,8$ & $827,5 \pm 192,7$ \\
\hline TOS & $49,44 \pm 27,14$ & $25,24 \pm 18,83$ \\
\hline
\end{tabular}

peroxide levels are dependent on the type of arthritis. The $\mathrm{LOOH}$ and lipofuscin concentrations in healthy children as compared with a group of children with JIA differ. Higher values occur in the acute forms of JIA, but the difference is not statistically significant. There is no correlation in the total oxidative status (TOS) and the inflammation's level in the joints.

\section{Disclosure of interest}

None declared.

\section{Authors' details}

${ }^{1}$ Department of Pediatrics, Zabrze, Poland. ${ }^{2}$ Medical University of Silesia, Zabrze, Poland. ${ }^{3}$ Department of Biochemistry, Medical University of Silesia, Zabrze, Poland.

Published: 5 December 2013

\section{doi:10.1186/1546-0096-11-S2-P70}

Cite this article as: Mazur Zielinska et al:: PReS-FINAL-2058: Lipid peroxides, lipophuscin, sulfhydryl groups and TOS in children with JIA. Pediatric Rheumatology 2013 11(Suppl 2):P70.

${ }^{1}$ Department of Pediatrics, Zabrze, Poland

Full list of author information is available at the end of the article 